\title{
ESTUDO EVOLUTIVO DA DOENÇA DE CHAGAS COM FASE AGUDA CONHECIDA
}

\author{
Humberto de Oliveira Ferreira *e Helio Pucci * *
}

Tendo sido feito um estudo evolutivo da doença de Chagas em 32 casos com fase aguda conhecida, sendo de oito anos o tempo médio de evolução da moléstia, os autores encontraram Guerreiro-Machado reagente em $71,2 \%$ dos pacientes, xeno-diagnóstico positivo em $25 \%$, aperistalsis do esofago (grupo I/ em cinco casos, megacolon em 3, aumento da área cardiaca também em 3. O eletrocardiograma foi anormal em 6 casos. Alguns pacientes tratados com nitrofuranos tiveram muito boa evolução.

\section{INTRODUÇÃO}

Sob os auspícios da Organização Panamericana de Saúde, constituiu-se um grupo composto dos Prof. Aluizio Prata, Prof. Anis Rassi, Dr. João Carlos Pinto Dias, Prof. Humberto de Oliveira Ferreira, Prof. Helio Pucci e Prof. Vicente Amato Neto, com a finalidade de estudar a evolução da doença de Chagas com fase aguda conhecida.

Os pacientes foram estudados mediante realização de exame clínico, xenodiagnóstico, reação de Guerreiro-Machado, radiografias do tórax, esôfago, colons e eletrocardiograma. Neste trabalho são apresentados alguns dos resultados observados.

\section{MATERIAL E MÉTODOS}

São descritos nos Quadros I, la, lb, Ic e Id os 32 casos revistos, considerando-se dados de identificação, aspectos radiológicos do tórax, eletrocardiogramas antes dos tratamentos, bem como aspectos clínicos, xenodiagnóstico, reação de Guerreiro-Machado, radiografias do tórax, esôfago e colons; eletrocardiogramas após tratamento com Bayer 2502 (7 casos), Spirotrypan (4 casos), nitrofurzona (3 casos), furazolidona
(1 caso), NF-602 (3 casos), levofuraltadona (1 caso) e primaquina ( 2 casos), sendo 11 casos não tratados para a doença de Chagas.

\section{RESULTADOS}

Os resultados do presente estudo encontram-se referidos nos Quadros apresentados.

Nos estudo dos 32 casos com fase aguda conhecida de doença de Chagas, o tempo médio de evolução da doença foi de 8 anos, sendo a média atual da idade de 16 anos. A reação de Guerreiro-Machado foi reagente em 23 casos $(71,2 \%)$ e não reagente em $9(28,2 \%)$; o xenodiagnóstico foi negativo em 24 casos $(75 \%)$ e positivo em 8 casos (25\%). Observou-se aperistalse do esôfago (grupo I) em 5 casos, megacolon em 3 e aumento da área cardiaca em 3 casos. $O$ eletrocardiograma foi normal em 15 casos, considerado dentro dos limites da normalidade em 11 e anormal em 6; nestes casos, observaram-se alteração difusa de repolarização ventricular em 3 casos, sistole elétrica prolongada em 1, sobrecarga ventricular esquerda em 1 e BVA do 19 grau em 1 caso.

Observou-se que alguns casos tratados na fase aguda com nitrofuranos tiveram boa evolução.

\footnotetext{
* Prof. Titular de Clínica Pediátrica Médica e Higiene Infantil da Faculdade de Medicina do Triângulo Mineiro.

* * Prof. Adjunto de Clínica Médica da Faculdade de Medicina do Triângulo Mineiro.
} 
Quadro la

Evolução da doença de Chagas com fase aguda conhecida

\begin{tabular}{|c|c|c|c|c|c|c|c|c|c|c|c|c|c|c|c|c|c|}
\hline \multirow{3}{*}{$\begin{array}{c}\text { Caso } \\
\text { N. }\end{array}$} & \multirow{3}{*}{ Iniciais } & \multirow{3}{*}{ Idade } & \multirow{3}{*}{ Sexo } & \multirow{3}{*}{$\begin{array}{c}\text { Fase } \\
\text { Aguda } \\
\text { (data) }\end{array}$} & \multicolumn{4}{|c|}{ Fase Aguda } & \multicolumn{9}{|c|}{ Revisão } \\
\hline & & & & & & $\mathrm{ECG}$ & Tratamento & & & $\frac{\mathrm{g}}{\mathrm{s}}$ & Xeno & Reação de & & $R x$ & & E.C.G. & ORS \\
\hline & & & & & $\begin{array}{c}\text { do } \\
\text { Torax }\end{array}$ & & & (Data) & $\begin{array}{l}\text { após fa- } \\
\text { se aguda }\end{array}$ & $\begin{array}{l}\bar{u} \\
\dot{x} \\
\end{array}$ & $\cdot$ & $\begin{array}{c}\text { reiro } \\
\text { Machado }\end{array}$ & Torax & Esof & Colons & & \\
\hline 1 & M.N.S. & $6 m$ & $\mathrm{~F} 2$ & 23.09 .65 & AAC & $N$ & $\begin{array}{l}\text { Bayer } \\
2502\end{array}$ & 09.08 .67 & $2 a$. & $\mathbf{N}$ & Pos. & NR & $N$ & $N$ & pr & $\begin{array}{r}\text { Sistole } \\
\text { elétrica } \\
\text { prolongada }\end{array}$ & \\
\hline 2 & R.C. & $6 a$. & F & 02.09 .66 & $\mathbf{N}$ & $\begin{array}{r}\text { Sistole } \\
\text { eletrica } \\
\text { prolongada }\end{array}$ & $\begin{array}{r}\text { Bayer } \\
2502\end{array}$ & -10.10 .67 & $1 \mathrm{a}$. & $\mathbf{N}$ & Neg. & NR & $N$ & $N$ & Tac & $\begin{array}{c}\text { aquicardia } \\
\text { sinusal } \\
\text { Arritmia } \\
\text { sinusal }\end{array}$ & \\
\hline 3 & R.R.S. & $7 a$ & M & 18.05 .65 & $N$ & $\begin{array}{r}\text { Taquicardia } \\
\text { sinusal } \\
\text { Sistole elétri- } \\
\text { ca prolongada }\end{array}$ & $\begin{array}{l}\text { Bayer } \\
2502\end{array}$ & 07.11 .67 & $2 \mathrm{a} .6 \mathrm{~m}$ & $N$ & Neg. & NR & $N$ & $N$ & $N$ & $N$ & \\
\hline 4 & N.T.O. & $11 \mathrm{a}$. & F. & 02.03 .65 & $\mathbf{N}$ & $\begin{array}{r}\text { Bloqueio AV } \\
19 \text { Grau } \\
\text { Alt.Dif.repol. }\end{array}$ & $\begin{array}{r}\text { Bayer } \\
2502\end{array}$ & 15.08 .67 & $2 \mathrm{a} .5 \mathrm{~m}$ & $\mathbf{N}$ & Neg. & NR & $N$ & $\mathbf{N}$ & $N$ & $\mathbf{N}$ & \\
\hline 5 & V.s.o. & $5 a$. & $F$ & 24.12 .65 & $N$ & $\begin{array}{r}\text { Taquicardia } \\
\text { sinusal } \\
\text { Sistole elétri- } \\
\text { ca prolongada }\end{array}$ & Bayer & 07.11 .67 & $2 a$. & $\mathbf{N}$ & Pos. & $\begin{array}{r}R \\
2502\end{array}$ & $N$ & $\mathbf{N}$ & $N$ & $N$ & \\
\hline 6 & M.A.L. & $13 a$ & $F$ & 24.09 .66 & $N$ & $\begin{array}{l}\text { Sistole elétri- } \\
\text { ca prolongada } \\
\text { Alt. rep. vent. } \\
\text { Isq. sub. epic. }\end{array}$ & $\begin{array}{l}\text { Bayer } \\
2502 \\
\end{array}$ & 09.11 .67 & $1 \mathrm{a} .3 \mathrm{~m}$ & $\mathbf{N}$ & Pos. & $\begin{array}{c}\mathbf{R} \\
>2.3 \\
\end{array}$ & $N$ & $\mathbf{N}$ & $N$ & $\begin{array}{l}\text { Arritmia } \\
\text { sinusal }\end{array}$ & \\
\hline 7 & M.C. 1 & $0.4 m$ & $F$ & 26.11 .52 & - & - & - & 04.03 .67 & $16 a$ & $N$ & Neg. & $\begin{array}{c}R \\
>3,3\end{array}$ & $N$ & $\mathbf{N}$ & $N$ & $\begin{array}{l}\text { BRD in- } \\
\text { completo }\end{array}$ & \\
\hline 8 & M.A.C. & $13.6 m$ & $\mathbf{F}$ & 18.10 .60 & - & - & $\begin{array}{l}\text { Spiro- } \\
\text { trypan }\end{array}$ & 23.03 .67 & $7 a$. & $\mathbf{N}$ & Neg. & NR. & $N$ & $\mathbf{N}$ & $\mathrm{Ba}$ & $\begin{array}{l}\text { aixa volta- } \\
\text { de QRS }\end{array}$ & $\begin{array}{r}\text { Tratado } \\
\text { Bayer } \\
2502\end{array}$ \\
\hline
\end{tabular}


Quadro lb

Evolução da doença de Chagas com fase aguda conhecida

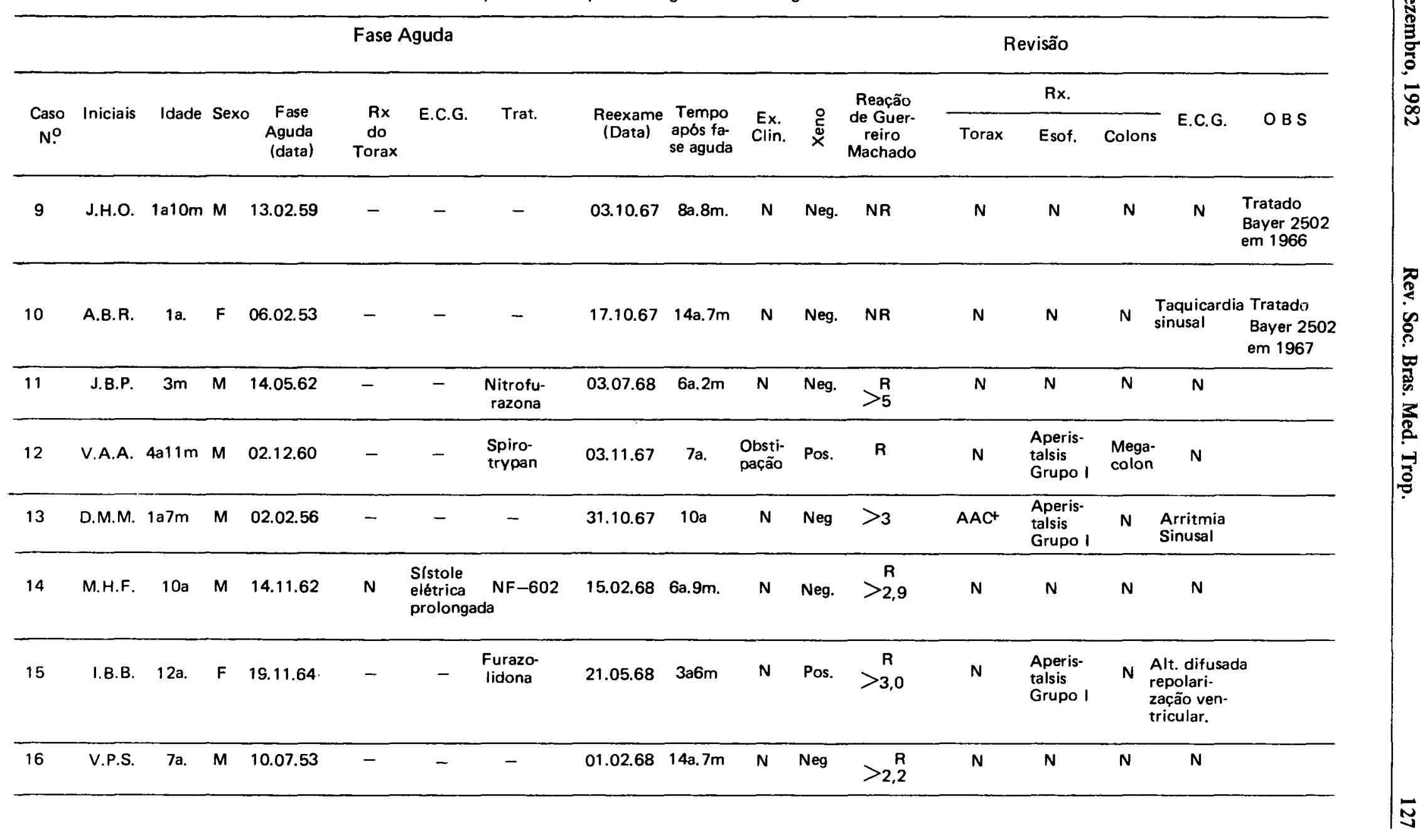


Quadro Ic

Evolução da doença de Chagas com fase aguda conhecida

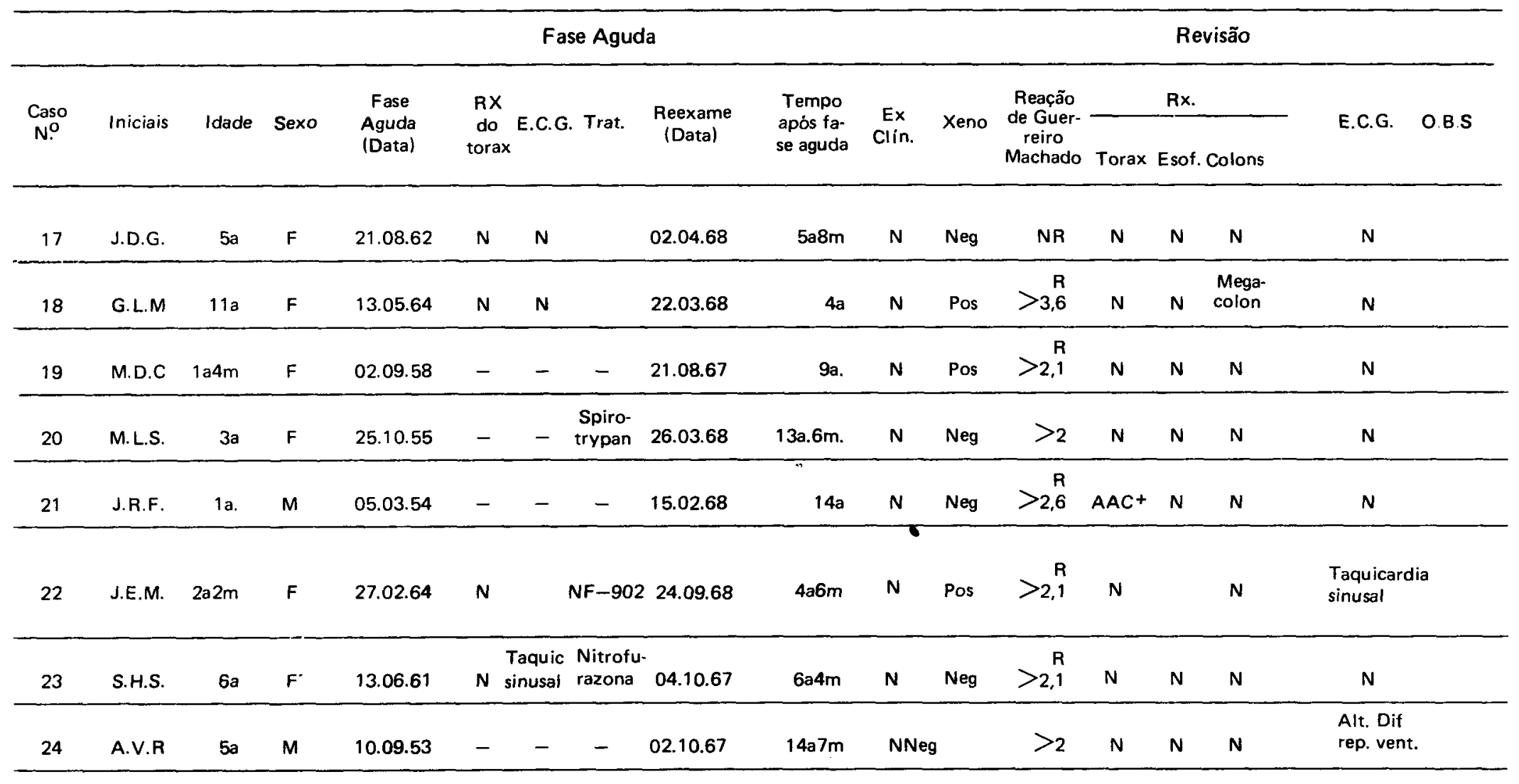


Quadro Id

Evolução da doença de Chagas com fase aguda conhecida

\begin{tabular}{|c|c|c|c|c|c|c|c|c|c|c|c|c|c|c|c|c|c|}
\hline \multirow{3}{*}{$\begin{array}{c}\text { Caso } \\
\mathrm{N}^{0}\end{array}$} & \multirow{3}{*}{ Iniciais } & \multirow{3}{*}{ Idade } & \multirow{3}{*}{ Sexo } & \multicolumn{5}{|c|}{ Fase aguda } & \multirow{3}{*}{$\begin{array}{l}\text { Tempo } \\
\text { após fa- } \\
\text { se aguda }\end{array}$} & \multirow{3}{*}{$\begin{array}{c}\text { Ex. } \\
\text { Clínica }\end{array}$} & \multirow{3}{*}{ Xeno } & \multirow{3}{*}{$\begin{array}{l}\text { Reação } \\
\text { de Guer- } \\
\text { reiro } \\
\text { Machado }\end{array}$} & \multicolumn{3}{|c|}{ Revisão } & \multirow{3}{*}{ E.C.G. } & \multirow{3}{*}{ O B S } \\
\hline & & & & \multirow{2}{*}{$\begin{array}{l}\text { Fase } \\
\text { Aguda } \\
\text { (Data) }\end{array}$} & \multirow{2}{*}{$\begin{array}{l}R X \\
\text { do } \\
\text { torax }\end{array}$} & \multirow{2}{*}{ E.C.G. } & \multirow{2}{*}{ 3. Trat. } & \multirow{2}{*}{$\begin{array}{c}\text { Reexame } \\
\text { (Data) }\end{array}$} & & & & & & $R x$. & & & \\
\hline & & & & & & & & & & & & & Torax & Esof. & Colons & & \\
\hline 25 & N.L.S. & $6 a$. & $M$ & 19.09.62 & - & - & $N F-602$ & 25.09 .67 & $5 a$. & $N$ & $\mathrm{Neg}$ & $\begin{array}{l}R \\
K 3\end{array}$ & $A A C^{++}$ & $+N$ & $N$ & \multicolumn{2}{|l|}{$\begin{array}{l}\text { Bradicardia } \\
\text { sinusal } \\
\text { S.V.E. } \\
\text { provável }\end{array}$} \\
\hline 26 & J.V.R. & $7 a$ & $\mathbf{F}$ & 05.09 .54 & - & - & - & 27.09 .67 & $13 a$. & $\mathbf{N}$ & $\mathrm{Neg}$ & $>2$ & $N$ & $N$ & $N$ & $\begin{array}{l}\text { Bradicardia } \\
\text { sinusal } \\
\text { Alt. RV }\end{array}$ & \\
\hline 27 & J.V.C & $11 a$ & $\mathbf{F}$ & 05.05 .51 & - & - & $\begin{array}{l}\text { Prima- } \\
\text { quina }\end{array}$ & 16.09 .67 & $16 a 4 m$ & $\begin{array}{l}\text { Obsti- } \\
\text { pação }\end{array}$ & Neg & $\stackrel{R}{P}_{2,1}$ & $N$ & $N$ & $\begin{array}{l}\text { Mega- } \\
\text { colon }\end{array}$ & $N$ & \\
\hline 28 & V.H.D. & $5 a$ & $F$ & 23.02 .60 & - & - & $\begin{array}{l}\text { Spiro- } \\
\text { trypan }\end{array}$ & 24.08 .67 & $7 a .6 m$ & $N$ & Neg & $\stackrel{R}{P}_{3}$ & $\mathbf{N}$ & $N$ & $N$ & $\begin{array}{l}\text { Bloqueio } \\
A V-1{ }^{\circ} \text { gráu }\end{array}$ & \\
\hline 29 & J.G.A & $1 a$ & $M$ & 02.05 .61 & - & - & - & 03.12 .67 & $6 a 7 m$ & $N$ & Neg & $\stackrel{R}{>}_{2,3}$ & $\mathbf{N}$ & $N$ & $N$ & & \\
\hline 30 & J.A.S. & $53 a$ & $M$ & 30.12 .66 & $\mathbf{N}$ & - & $\begin{array}{l}\text { Bayer } \\
2502\end{array}$ & 07.06 .68 & $1 \mathrm{a} .6 \mathrm{~m}$ & $\mathbf{N}$ & Neg & NR & $N$ & $N$ & $N$ & $\begin{array}{l}\text { Bradicardia } \\
\text { sinusal }\end{array}$ & \\
\hline 31 & A.F.L. & $41 a$ & $M$ & 15.03 .53 & - & - & - & 20.08 .68 & $15 a .5 m$ & $n \quad N$ & Neg & $\begin{array}{l}R \\
5\end{array}$ & t & $\begin{array}{l}\text { Aperis- } \\
\text { talsis } \\
\text { Grupo I }\end{array}$ & $N$ & $\begin{array}{l}\text { Taquicardia } \\
\text { sinusal }\end{array}$ & \\
\hline 32 & M.J.B & $27 a$ & $M$ & 14.02 .52 & - & - & $\begin{array}{l}\text { Prima- } \\
\text { quina }\end{array}$ & 14.09 .67 & $15 \mathrm{a} .7 \mathrm{~m}$ & $n N$ & Neg. & $\stackrel{R}{>}$ & & $\begin{array}{l}\text { Aperis- } \\
\text { talsis } \\
\text { Grupo I }\end{array}$ & & $\begin{array}{l}\text { Baixa vol- } \\
\text { tagerm de } \\
\text { QRS }\end{array}$ & \\
\hline
\end{tabular}




\section{SUMMARY}

As an evolutive study of Chagas disease has been carried out in 32 patients diagnosed in acute phase, and followed-up for 8 years (medium time of the evolution of the disease). The authors found Guerreiro-Machado reagent in $71,8 \%$ of the pacients, positive xenodiagnosis in $25 \%$, aperistalsis of the esophagus in 5 cases and megacolon in 3 cases; and also dilation of the cardiac are in 3 pacients.

The eletrocardiography was abnormal in 6 cases.

Some pacients who were submited to treatment wild nitrofuranic during the acute phase compounds during the acute phase had a very good evolution. 